\title{
1 Signaling from the RNA sensor RIG-I is regulated by ufmylation
}

6 Daltry L. Snider ${ }^{1}$, Moonhee Park ${ }^{1}$, Kristen A. Murphy ${ }^{1}$, Dia C. Beachboard ${ }^{1, \text { a }}$, Stacy M.

7 Horner $^{1,2, *}$

$11{ }^{1}$ Department of Molecular Genetics \& Microbiology, Duke University Medical Center,

12 Durham, NC 27710, USA

$13{ }^{2}$ Department of Medicine, Duke University Medical Center Durham, NC 27710, USA

${ }^{\text {a }}$ Current address: DeSales University, Center Valley, Pennsylvania, 18034

* Correspondence: stacy.horner@duke.edu

20 Stacy M. Horner, Ph.D.

21 Duke University Medical Center

$22 \quad 213$ Research Dr., Box 3053 DUMC

23 Durham, NC USA 27710

\section{Keywords}

28 Retinoic acid-inducible gene I, RLR signaling, ubiquitin-like modifications, mitochondrial-

29 associated ER membranes 


\section{Abstract}

32 The RNA binding protein RIG-I is a key initiator of the antiviral innate immune response.

33 The signaling that mediates the antiviral response downstream of RIG-I is transduced

34 through the adaptor protein MAVS and results in the induction of type I and III interferons

35 (IFN). This signal transduction occurs at endoplasmic reticulum (ER)-mitochondrial 36 contact sites, to which RIG-I and other signaling proteins are recruited following their 37 activation. RIG-I signaling is highly regulated to prevent aberrant activation of this pathway 38 and dysregulated induction of IFN. Previously, we identified UFL1, the E3 ligase of the 39 ubiquitin-like modifier conjugation system called ufmylation, UFL1, as one of the proteins 40 recruited to membranes at ER-mitochondrial contact sites in response to RIG-I activation.

41 Here, we show that UFL1, as well as the process of ufmylation, promote IFN induction in 42 response to RIG-I activation. We find that following RNA virus infection, UFL1 is recruited

43 to the membrane targeting protein $14-3-3 \varepsilon$, and that this complex is then recruited to 44 activated RIG-I to promote downstream innate immune signaling. Importantly, loss of 45 ufmylation prevents $14-3-3 \varepsilon$ interaction with RIG-I, which abrogates the interaction of RIG46 I with MAVS and thus downstream signal transduction that induces IFN. Our results define 47 ufmylation as an integral regulatory component of the RIG-I signaling pathway and as a 48 post-translational control for IFN induction.

\section{Significance}

51 The viral RNA sensor RIG-I initiates the antiviral innate immune response by activating a 52 signaling cascade that induces interferon. Activation of the RIG-I signaling pathway is 53 highly regulated to quickly mount a protective immune response while preventing 54 dysregulation that can lead to excessive inflammation or autoimmune disorders. Here, we 55 characterize one such mechanism of regulation. We describe that UFL1, an E3 ligase for 56 the ubiquitin-like modifier conjugation system called ufmylation, is important to promote 57 RIG-I signaling. Using molecular approaches, we show that ufmylation promotes RIG-I 58 interaction with the membrane targeting protein 14-3-3e. As such, ufmylation positively 59 regulates RIG-I recruitment to its signaling adaptor proteins MAVS for induction of 60 interferon in response to RNA virus infection. 


\section{Abstract}

64 The RNA binding protein RIG-I is a key initiator of the antiviral innate immune response.

65 The signaling that mediates the antiviral response downstream of RIG-I is transduced 66 through the adaptor protein MAVS and results in the induction of type I and III interferons 67 (IFN). This signal transduction occurs at endoplasmic reticulum (ER)-mitochondrial 68 contact sites, to which RIG-I and other signaling proteins are recruited following their 69 activation. RIG-I signaling is highly regulated to prevent aberrant activation of this pathway 70 and dysregulated induction of IFN. Previously, we identified UFL1, the E3 ligase of the 71 ubiquitin-like modifier conjugation system called ufmylation, UFL1, as one of the proteins 72 recruited to membranes at ER-mitochondrial contact sites in response to RIG-I activation.

73 Here, we show that UFL1, as well as the process of ufmylation, promote IFN induction in 74 response to RIG-I activation. We find that following RNA virus infection, UFL1 is recruited 75 to the membrane targeting protein $14-3-3 \varepsilon$, and that this complex is then recruited to 76 activated RIG-I to promote downstream innate immune signaling. Importantly, loss of 77 ufmylation prevents $14-3-3 \varepsilon$ interaction with RIG-I, which abrogates the interaction of RIG78 I with MAVS and thus downstream signal transduction that induces IFN. Our results define 79 ufmylation as an integral regulatory component of the RIG-I signaling pathway and as a 80 post-translational control for IFN induction.

81 Significance Statement

82 The viral RNA sensor RIG-I initiates the antiviral innate immune response by activating a 83 signaling cascade that induces interferon. Activation of the RIG-I signaling pathway is 84 highly regulated to quickly mount a protective immune response while preventing 85 dysregulation that can lead to excessive inflammation or autoimmune disorders. Here, we 86 characterize one such mechanism of regulation. We describe that UFL1, an E3 ligase for 87 the ubiquitin-like modifier conjugation system called ufmylation, is important to promote 88 RIG-I signaling. Using molecular approaches, we show that ufmylation promotes RIG-I 89 interaction with the membrane targeting protein 14-3-3e. As such, ufmylation positively 90 regulates RIG-I recruitment to its signaling adaptor proteins MAVS for induction of 91 interferon in response to RNA virus infection.

92

\section{Introduction}

95 Detection of RNA virus infection is initiated by cellular sensors such as RIG-I. RIG-I is a 96 pattern recognition receptor that detects unique features of viral RNA that are generally 
97 absent in cellular RNA, referred to as pathogen-associated molecular patterns (PAMPs)

98 (1). Sensing of viral RNA PAMPs triggers RIG-I activation and induces a downstream 99 signaling cascade that ultimately results in transcriptional induction of type I and type III 100 interferons (IFN) and the antiviral response (2, 3). The RIG-I signaling cascade is carefully 101 regulated by multiple mechanisms, including post-translational modifications that 102 influence specific protein-protein interactions that can result in changes in protein 103 localization to mediated signaling $(3,4)$. For example, following sensing of RNA PAMPs, 104 RIG-I undergoes K63-linked polyubiquitination in order to transition to its fully active 105 conformation, which promotes its interaction with the molecular trafficking protein 14-3-3ع 106 (5-8). 14-3-3ع facilitates the recruitment of activated RIG-I from the cytosol to intracellular 107 membranes where it interacts with $\operatorname{MAVS}(7,9,10)$, which assembles other RIG-I pathway 108 members to transduce the signals that induce IFN $(7,11)$. Importantly, many RNA viruses, 109 including influenza A virus and some flaviviruses (dengue virus, Zika virus, and West Nile 110 virus), prevent the interaction of RIG-I with $14-3-3 \varepsilon$ to limit IFN induction and evade the 111 antiviral response $(9,10,12)$.

113 In addition to RIG-I, a number of signaling proteins must be recruited to MAVS in order to 114 propagate downstream IFN induction. Previously, we identified proteins that move to 115 MAVS signaling sites at mitochondrial-associated endoplasmic reticulum (ER) 116 membranes (MAM) during RNA virus infection $(13,14)$. These proteins likely aid in spatial 117 organization of RIG-I pathway proteins during viral infection and include the GTPase 118 RAB1B, which plays a role in recruiting TRAF3 to MAVS (15). In addition to RAB1B, we 119 identified other proteins recruited to the MAM upon RIG-I signaling activation, one of which 120 was UFL1 (referred to in our previous publication as KIAA0776) (14). UFL1 is an E3 ligase 121 for UFM1, which is a ubiquitin-like modification of 85 amino acids. The process of 122 ufmylation conjugates UFM1 covalently to lysine residues of target proteins through a 123 process called ufmylation, which is similar to ubiquitination in that it also uses an E1, E2, 124 and E3 ligase conjugation system (UBA5, UFC1, and UFL1; see Figure 2D). UFM1 is 125 removed by the UFSP2 protease (16-20). The consequence of UFM1 addition to proteins 126 is not fully understood, but the literature supports the idea that it can promote protein127 protein interactions to regulate a number of biological processes (21-31). Here, we 128 uncover a role for ufmylation in RIG-I activation. We found that the cellular proteins that 129 catalyze ufmylation all promote RIG-I-mediated induction of IFN. Interestingly, we found 130 that UFL1 interacts with both RIG-I and the molecular trafficking protein $14-3-3 \varepsilon$ following 
131 RNA virus infection. Further, similar to RIG-I, UFL1 is recruited to intracellular membranes

132 following RNA virus infection. Importantly, loss of ufmylation prevents the interaction of

133 14-3-3e with RIG-I, which results in decreased MAVS activation and IFN induction in 134 response to RNA virus infection. Thus, ufmylation can regulate RIG-I activation and 135 downstream signaling of the intracellular innate immune system.

\section{Results}

139 The ufmylation activity of UFL1 promotes RIG-I signaling. Having found that the E3 140 ligase of ufmylation UFL1 is recruited to MAVS signaling sites at the MAM in response to 141 RIG-I signaling (14), we wanted to determine if UFL1 regulates RIG-I signaling. To test 142 this, we measured induction of the IFN- $\beta$ promoter following UFL1 overexpression using 143 an IFN- $\beta$ promoter luciferase reporter assay (32) and found that UFL1 increased activation 144 of the IFN- $\beta$ promoter, similar to that of RIG-I expression, in a dose-dependent fashion in 145 response to infection with Sendai virus (SenV) (Figure 1A). SenV is a murine 146 paramyxovirus that specifically activates RIG-I (33). In support of UFL1 enhancing RIG-I 147 signaling specifically, exogenous expression of UFL1 also increased IFN- $\beta$ promoter 148 activity in response to transfection of 293T cells with a known RIG-I immunostimulatory 149 RNA from hepatitis C virus (PAMP; Figure 1B) (34). However, UFL1 overexpression in 150 293T cells did not lead to increased induction of IFN-stimulated genes (ISG), such as 151 ISG56 or ISG15, in response to exogenous IFN- $\beta$ treatment, indicating that UFL1 primarily 152 regulates IFN induction and not the IFN response (Figure 1C). Next, we depleted UFL1 153 by siRNA in two different cell types and measured SenV-induced activation of the RIG-I 154 pathway. Depletion of UFL1 in 293T cells resulted in decreased phosphorylation of IRF3, 155 a transcription factor for both type I and III IFNs, while exogenous expression of an siRNA156 resistant UFL1 restored SenV-mediated IRF3 phosphorylation (Figure 1D). Depletion of 157 UFL1 in primary neonatal human dermal fibroblasts (NHDFs) also reduced the SenV158 mediated induction of both IFNB1 and IFNL1 transcripts, as measured by RT-qPCR 159 (Figure 1E), as well as the production of IFN- $\beta$ protein, as measured by an enzyme-linked 160 immunosorbent assay (ELISA) (Figure 1F).

162 To define the domains of UFL1 that regulate RIG-I signaling, we expressed a series of 163 previously described UFL1 truncation mutants (diagrammed in Figure $1 \mathrm{H}$ ) and measured 164 SenV-mediated activation of the IFN- $\beta$ promoter in a luciferase reporter assay (16). The 
ability of UFL1 to transfer UFM1 to a target protein has been suggested to require the first 212 amino acids of the protein, as this domain interacts with the E2 ligase for ufmylation, UFC1 (16). The wild-type (WT) UFL1 (aa 1-794), as well as the C-terminal deleted mutants of UFL1, aa 1-212 and aa 1-452, which all have reported ufmylation activity (16), stimulated SenV-medicated induction of the IFN- $\beta$ promoter (Figure 1G). Interestingly, the $\mathrm{N}$-terminal deleted mutant of UFL1 aa 213-794, that does not have reported ufmylation activity, also induced signaling, while the N-terminal deleted UFL1 mutant aa 453-794 did not (Figure 1G). However, our analysis of global UFM1 conjugates by these UFL1 constructs revealed that while UFL1 WT, aa 1-212, and aa 1-452 all retain full ufmylation activity, aa $213-794$ of UFL1 retain approximately $40 \%$ ufmylation activity, while aa $453-$ 794 of UFL1 retain only about $20 \%$ activity (Figure $1 \mathrm{H}$; Figure S1). Thus, taken together, this reveals that the ufmylation activity of UFL1 is required to promote RIG-I signaling that results in induction of IFN.

The ufmylation machinery proteins positively regulate RIG-I signaling. Having determined that the ufmylation activity of UFL1 is important for its role in RIG-I signaling, we hypothesized that UFM1 and the proteins required for UFM1 conjugation would also be required to promote this signaling. Similar to our results with UFL1, overexpression of UFM1 increased SenV-mediated activation of the IFN- $\beta$ promoter in a dose-dependent fashion (Figure 2A). Conversely, the activation of the IFN- $\beta$ promoter in response to SenV was significantly abrogated in 293T cells in which UFM1 was deleted by CRISPR/Cas9, as compared to WT 293T cells (Figure 2B). Importantly, this signaling was restored upon exogenous expression of UFM1 (Figure 2B). The absence of UFM1 expression also prevented the induction of IFN- $\beta$ protein in response to SenV infection, as measured by ELISA (Figure 2C). The process of ufmylation has 5 steps (Figure 2D). First, UFM1 is processed to expose the terminal glycine residue. Then, this mature UFM1 is added to the target protein by the actions of UBA5, which acts as an E1 ligase for UFM1; UFC1, the E2 ligase; and UFL1, the E3 ligase (19). Finally, the UFSP2 protease removes UFM1, which enables recycling of mature UFM1 (18). We found that exogenous expression of each of

194 the proteins involved in UFM1 conjugation, including the UFSP2 protease, positively 195 regulated SenV-mediated induction of the IFN- $\beta$ promoter (Figure 2E). These results 196 reveal that the proteins that catalyze ufmylation and the UFM1 modification itself promote 197 RIG-I signaling. 
UFM1 is required for the RIG-I-driven transcriptional response. After establishing that ufmylation promotes RIG-I activation, and in turn IFN expression, we next broadly measured the impact of ufmylation upon the transcriptional response to RIG-I signaling. Using RNA-sequencing, we analyzed gene expression in either WT or UFM1 KO 293T cells, following mock or SenV infection (Table S1.1; Table S1.2). Gene set enrichment analysis (Table S2.1; Table S2.2) of the transcripts significantly reduced (adjusted $P<0.01$ ) by UFM1 KO in the absence of viral infection revealed previously described pathways regulated by ufmylation such as cytosolic ribosomes, ribosome assembly, and

207 hematopoiesis (Figure S2A; Table S2.1) (21, 28, 29, 35). Following viral infection, the top

20810 gene categories negatively impacted by UFM1 KO, with a darker red color indicating more downregulation, were all related to the antiviral response, such as response to type I IFN and defense against virus (Figure 3A; Table S2.2). Indeed, of the top 50 most downregulated pathways impacted by UFM1 KO during infection, the majority were related to innate immune signaling or viral replication (Table S2.2), while upregulated gene categories were more diverse (Table S2.3; Table S2.4). Of the genes differentially expressed during UFM1 KO in response to SenV (adjusted $\mathrm{P}<0.01$ ), the majority are downregulated (Figure 3B). Indeed, these downregulated genes included IFNB1 and IFNL1, as well as other known ISGs (in red) (36) (Figure 3B; Figure 3C). These data are consistent with a model in which ufmylation-mediated regulation of IFN induction has broad consequences on genes induced by the IFN response.

UFL1 is recruited to intracellular membranes and interacts with $14-3-3 \varepsilon$ and RIG-I during RNA virus infection. Following the binding of RIG-I to non-self RNA, it interacts with several host proteins to facilitate its activation, localization to the MAM, and interaction with MAVS. These proteins include the E3 ligases for K63-linked ubiquitin TRIM25 and

224 Riplet $(5,6,37)$, as well as the molecular trafficking protein 14-3-3e. In particular, 14-3-3e

225 is required for RIG-I recruitment from the cytosol to MAVS signaling sites at intracellular membranes (5-7, 13); however, the mechanism underlying how $14-3-3 \varepsilon$ selects RIG-I as cargo has yet to be elucidated. Using a subcellular membrane fractionation assay (38), we confirmed that UFL1 increases its association with intracellular membranes in response to SenV, similar to RIG-I (Figure 4A; compare fraction \#1, which has Cox-I and no GAPDH, with fractions \#6-8, which are enriched for the cytosolic protein GAPDH) (7,

231 12). This finding is consistent with our previous report that UFL1 is recruited to the MAM 232 in response to either SenV or hepatitis C virus replication (14), suggesting that UFL1 
233 recruitment occurs prior to MAVS activation, as MAVS is cleaved by the HCV NS3-NS4A

234 protease (39-42). As the recruitment of RIG-I to intracellular membranes is known to

235 require $14-3-3 \varepsilon$, and, as both UFL1 and UFM1 have been shown to interact with 14-3-3ع

236 (16), we hypothesized that UFL1 may interact with 14-3-3 $\varepsilon$ to promote the IFN induction

237 that we had observed in response to RNA virus infection. Thus, we first determined if the

238 interaction of UFL1 with 14-3-3 $\varepsilon$ is increased in response to RIG-I activation by SenV by

239 performing co-immunoprecipitation. We found that Myc-14-3-3e did co-immunoprecipitate

240 with Flag-UFL1, as reported previously (16), and that this interaction was increased by

241 SenV (Figure 4B). Interestingly, the interaction of UFL1 with RIG-I also increased following

242 SenV, both upon over-expression and at the level of the endogenous proteins (Figure 4C;

243 Figure 4D). As RIG-I undergoes a series of modifications to become fully active (1, 4), we

244 next used a panel of RIG-I mutants to define which stage of RIG-I activation promotes

245 interaction with UFL1. These mutations prevent the distinct steps of RIG-I activation such

246 as RIG-I binding to RNA (K888/907A), interacting with TRIM25 (T55I), or ubiquitination by

247 Riplet and TRIM25 (K172/788R) (5, 43, 44). The interaction of UFL1 with RIG-I was

248 significantly impaired by each of these mutations, suggesting that UFL1 regulates RIG-I

249 function after it binds RNA and becomes ubiquitinated (Figure 4E). As this is the same

250 step of activation at which $14-3-3 \varepsilon$ binds to RIG-I to promote its translocation to

251 intracellular membranes (7), this suggests that RNA virus infection increases the

252 interaction of $14-3-3 \varepsilon$ with UFL1, which then interacts with activated, K63-ubiquitinated

253 RIG-I.

254

255 UFL1 interaction with RIG-I requires 14-3-3 $\varepsilon$ and UFM1. Having determined that UFL1

256 interacts with both activated RIG-I and $14-3-3 \varepsilon$ following RNA virus infection, we next

257 defined the dynamics of this complex formation by testing two distinct models. In the first

258 model, UFL1 would interact first with activated RIG-I, induce its ufmylation, and then the

259 UFL1-RIG-I complex would interact with 14-3-3e. In this model, depletion of $14-3-3 \varepsilon$ or

260 loss of UFM1 would not be expected to change the interaction of UFL1 with RIG-I. In the

261 second model, UFL1 would interact first with 14-3-3e and induces its ufmylation, or that of

262 another associated protein, and then the UFL1-14-3-3e complex would interact with

263 activated RIG-I. In this second model, depletion of 14-3-3e would be expected to prevent

264 UFL1 interaction with RIG-I, and loss of ufmylation would limit UFL1 interaction with RIG-

265 I but would not affect UFL1 interaction with 14-3-3ع. To elucidate these possibilities, first, 
266 we used co-immunoprecipitation to measure the interaction of exogenously expressed

267 Flag-UFL1 and HA-RIG-I in SenV-infected 293T lysates that had been depleted of 14-3-

$2683 \varepsilon$ or CTRL by siRNA. This revealed that formation of the SenV-activated RIG-I-UFL1

269 complex requires $14-3-3 \varepsilon$ (Figure 5A). Next, we tested if ufmylation was required for

270 formation of the SenV-activated RIG-I-UFL1 complex by measuring this interaction in WT

271 or UFM1 KO 293T cells. We found that UFM1 was required for SenV-activated RIG-I-

272 UFL1 complex (Figure 5B). The results of these two experiments reveal that both 14-3-3ع

273 and UFM1 are required for UFL1 to interact with RIG-I, supporting the second model of

274 complex formation in which UFL1 interacts first with 14-3-3 $\varepsilon$ and catalyzes its ufmylation,

275 and then this complex associates with RIG-I. In support of this, we found that UFM1 was

276 not required for UFL1 to interact with 14-3-3ع (Figure 5C). Together, these data indicate

277 that ufmylation promotes the interaction of UFL1 with 14-3-3e and activated RIG-I.

278

279 Ufmylation promotes RIG-I interaction with 14-3-3e for MAVS activation. Having 280 found that that UFL1 requires $14-3-3 \varepsilon$ to interact with activated RIG-I, we next tested if 281 UFL1 is required for the interaction of $14-3-3 \varepsilon$ with RIG-I, which is essential for activated 282 RIG-I to translocate from the cytosol to intracellular membranes for interaction with MAVS 283 (7). We performed a co-immunoprecipitation of Flag-RIG-I and Myc-14-3-3e from 293T 284 cells and found that this SenV-mediated interaction was significantly decreased upon 285 UFL1 depletion (Figure 6A). In addition, loss of UFM1 expression also decreased the 286 SenV-induced interaction of RIG-I with 14-3-3ع (Figure 6B). Importantly, we also found 287 that UFM1 is required for the SenV-induced interaction of RIG-I with MAVS (Figure 6C) 288 and MAVS higher-order oligomerization, which is a hallmark of MAVS activation $(45,46)$ 289 (Figure 6D). In summary, these data reveal that UFL1 and UFM1 are required for the RIG290 I interaction with 14-3-3e, for interaction with MAVS, and for MAVS activation by 291 oligomerization.

292

\section{Discussion}

295 Regulation of RIG-I activation and downstream signaling is essential for proper induction 296 and termination of IFN. Here, we show that both UFL1 and the process of ufmylation 297 promote RIG-I pathway signaling that leads to IFN induction, uncovering an important step 298 in the activation of the RIG-I pathway. RIG-I activation occurs upon RNA binding. Then, 
RIG-I undergoes ATP hydrolysis. and interaction with K63-linked polyubiquitin chains, both covalently and non-covalently $(5,44,47)$, which promotes formation of a RIG-I tetramer (48). This polyubiquitinated, activated RIG-I oligomer then interacts with the membrane trafficking protein $14-3-3 \varepsilon$ for translocation to MAVS at ER-mitochondrial contact sites (7). We found that UFL1 is recruited to $14-3-3 \varepsilon$ following RNA virus infection and that ufmylation facilitates the interaction between 14-3-3e and activated RIG-I. Importantly, this results in increased interaction of RIG-I with MAVS and MAVS oligomerization, ultimately promoting the downstream signal transduction which produces IFN.

309 Ufmylation is emerging as a post-translational modification that regulates diverse 310 biological processes, including DNA repair, ER homeostasis, and even the replication of 311 hepatitis A virus $(21,22,24,27,28,30,35,49)$. In these cases, UFL1, along with the other 312 members of the ufmylation cascade, induce ufmylation of a target protein important for 313 regulating these processes. For example, both MRE11 and histone $\mathrm{H} 4$ are ufmylated by 314 UFL1 in the nucleus in response to DNA damage resulting in activation the key DNA repair 315 kinase $\operatorname{ATM}(22,24)$. UFL1 can also act at the ER, where it plays a role in ER protein 316 quality control, where it ufmylates specific proteins, including ribosomal proteins RPL26, 317 to induce lysosomal degradation of stalled peptides and/or the ER and prevent the 318 unfolded protein response $(27,28,49,50)$. Interestingly, hepatitis A virus translation, 319 which occurs in association with the ER, also requires ufmylation of RPL26 (30). 320 Therefore, ufmylation can regulate several aspects of translation. It is possible that 321 ufmylation regulates translation of certain mRNAs important for RIG-I signaling and 322 subsequent IFN induction. However, we identified a role for ufmylation in regulating the 323 interaction of RIG-I with 14-3-3e, one of the earliest known steps of RIG-I signaling, 324 strongly supporting a mechanism in which following RIG-I activation, ufmylation is 325 controlling this specific protein-protein interaction. The mechanisms by which the process 326 of UFM1 addition regulates interactions between proteins or alters other aspects of protein 327 function are largely unknown. Indeed, we found that UFSP2, the protease that removes 328 UFM1 from proteins (18), promoted SenV-mediated IFN induction (Figure 2D), suggesting 329 that we do not yet have a full grasp on the ufmylation process. It is possible that the 330 dynamic process of ufmylation or the enhanced formation of mature UFM1 following 331 deconjugation from targets or promote RIG-I signaling independent of deconjugation 332 activity. Indeed, in support of this idea, others have shown that UFSP2 in myeloid cells is 
333 required for influenza virus resistance in mice (31). It is also possible that UFSP2 acts on

334 other members of the RIG-I pathway to alter their function. Future studies to define how

335 the process of ufmylation regulates this and other aspects of the antiviral innate immune

336 response will be of great interest as they may shed light broadly on how ufmylation

337 regulates diverse cell biological processes that alter cellular signaling.

The mechanisms underlying how cytoplasmic UFL1 is recruited to its protein targets that reside in different subcellular compartments are not fully known. For example, we found that RIG-I activation induces UFL1 translocation to intracellular membranes (Figure 3A), and while we know that UFL1 is recruited to the MAM during infection, the mechanism by which UFL1 becomes membrane-associated remains unknown (14). DDRGK1 (UFBP1) may facilitate UFL1 targeting to the MAM, as DDRGK1 is localized to mitochondrial-ER contact sites $(16,51)$ and in some cases it is required for UFL1 recruitment to membranes $(27,28)$. Thus, both DDRGK1 and mitochondrial-ER contact sites could function as a regulatory hub that aids in the recruitment of UFL1 and RIG-I pathway signaling proteins. Interestingly, RAB1B, a GTPase that we found is recruited to the MAM and important for RIG-I signaling $(14,15)$ is ufmylated $(52,53)$, which reveals that ufmylation likely regulates a number of RIG-I pathway signaling proteins. As UFL1 contains no functional domains common to other E3 ligases that might allow one to predict how its targets are selected $(16,54,55)$, defining the signals and features that control UFL1 localization, as well as the target proteins ufmylated in response to RIG-I activation, will undoubtedly reveal clues into how the process of ufmylation is activated and how specific targets are selected.

Our work revealed that $14-3-3 \varepsilon$ required ufmylation to interact with activated RIG-I. The details underlying how $14-3-3 \varepsilon$ interacts with activated RIG-I have not been fully elucidated, as it does not occur through the known phosphorylated amino acids on RIG-I, the typical recruitment signal of the 14-3-3 family of proteins $(7,56,57)$. Interestingly,

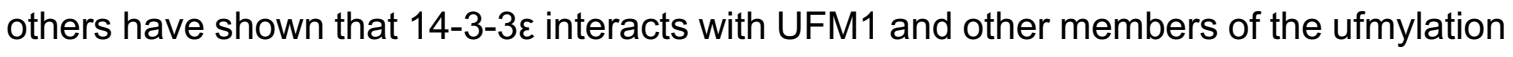

$3623 \varepsilon$ or a $14-3-3 \varepsilon$-associated protein promotes the interaction between activated RIG-I and 363 14-3-3 $\varepsilon$. In fact, a number of 14-3-3 family proteins are post-translationally modified by 364 phosphorylation, acetylation, and oxidation (58). Therefore, post-translational modification 365 of $14-3-3 \varepsilon$ by ufmylation could broadly define how cargo proteins, including RIG-I, are 366 selected. Indeed, this mechanism could be shared with other RNA virus sensing 
pathways, such as the RIG-I-like-receptor MDA5, which also interacts with a 14-3-3

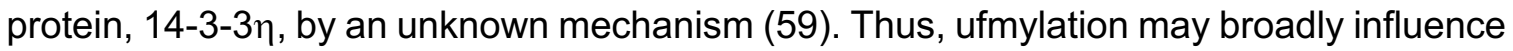
how 14-3-3 proteins or other host proteins interact with each other to regulate the

370 intracellular innate immune response.

372 Overall, this work lays the groundwork for future studies to define how ufmylation of 373 antiviral innate immune signaling proteins regulates their function and how specific 374 signaling pathways are differentially activated through ufmylation. In addition, our work 375 adds ufmylation to the growing list of ubiquitin-like and other modifications that regulate 376 the intracellular innate immune response, including ISGylation, SUMOylation, FATylation, 377 acetylation, phosphorylation, and others $(4,60,61)$ broadening our understanding of how 378 RIG-I signaling is activated and rapidly controlled by post-translational modifications in response to infection, leading to greater knowledge of the exquisite regulation of these pathways.

Materials and Methods

Cell lines, viruses, and treatments. Neonatal human dermal fibroblast (NHDF) cells and embryonic kidney 293T cells were grown in Dulbecco's modification of Eagle's medium (DMEM; Mediatech) supplemented with 10\% fetal bovine serum (Thermo Fisher Scientific), 1X minimum essential medium non-essential amino acids (Thermo Fisher Scientific), and $25 \mathrm{mM}$ HEPES (Thermo Fisher Scientific) (cDMEM). 293T (CRL-3216) were obtained from American Type Culture Collection (ATCC), NHDF cells (CC-2509) were obtained from Lonza. All cell lines were verified as mycoplasma free by the LookOut Mycoplasma PCR detection kit (Sigma). SenV Cantell strain was obtained from Charles

392 River Laboratories and used at 200 hemagglutination units/mL (HAU). SenV infections

393 were performed in serum-free media (30 minutes to 1 hour), after which complete media

394 was replenished. IFN- $\beta$ (PBL Assay Science) was added to cells at a concentration of 50 395 units/mL in cDMEM for 18 hours.

397 Plasmids. The following plasmids have been previously described: pEF-TAK-Flag, pEF398 BOS-Flag-RIG-I (62), pIFN- $\beta$-luc (63), pCMV-Renilla (Promega), pX459 (Addgene 399 Plasmid \#62988), pEF-BOS-Flag-RIG-I T55I (64), pEF-TAK-Myc-MAVS (32). 400 pLJM1_Flag-UFM1 was a gift from Dr. Craig McCormick at Dalhouise University. The 
401 following plasmids were generated by insertion of PCR-amplified fragments into the Notl-

402 to-Pmel digested pEF-TAK-Flag using InFusion cloning (Clontech), all primers are 403 denoted in Table 1: pEF-TAK-Flag-UFL1 (GenBank: BC036379; GenelD: 23376), pEF404 TAK-Flag-UBA5 (NM_024818.6), pEF-TAK-Flag-UFC1 (NM_016406.4), pEF-TAK-Flag405 UFSP2 (NM_018359.5), pEF-TAK-Flag-UFL1 1-212, pEF-TAK-Flag-UFL1 1-452, pEF406 TAK-Flag-UFL1 213-794, and pEF-TAK-Flag-UFL1 453-794. Both pEF-TAK-Myc-14-3-3ع 407 and pEF-TAK-Myc-UFL1 were generated by insertion of PCR-amplified fragments into the 408 Agel-Notl digested pEF-TAK-Myc (pEF-TAK-Myc-MAVS) by InFusion. The pEF-TAK-HA 409 vector was generated by PCR to replace Flag with HA, and pEF-TAK-HA-RIG-I was 410 generated by insertion of a PCR-amplified fragment into the Notl-Agel digested pEF-TAK411 HA vector. The following plasmids were generated by site-directed mutagenesis (see 412 Table 1): pEF-TAK-Flag-UFL1 ${ }^{\text {siR }}$, pEF-BOS-Flag-RIG-I K888/907A, and pEF-BOS-Flag413 RIG-I K172/788R. To generate the CRISPR guide RNA plasmids px459-UFM1-E2 and 414 px459-UFM1-B, sgRNA oligonucleotides were annealed and inserted into the Bbsl415 digested pX459 $(30,65)$. The plasmid sequences were verified by DNA sequencing and 416 oligonucleotide sequences are available upon request.

418 Generation of RNA PAMP. Annealed oligonucleotides containing the sequence of the 419 HCV 5'ppp poly-U/UC region (34) were in vitro transcribed using the MEGAshortscript T7 420 transcription kit (Ambion) followed by ethanol precipitation, with the resulting RNA 421 resuspended at $1 \mu \mathrm{g} / \mu \mathrm{L}$.

423 Transfection. DNA transfections were performed using FuGENE6 (Promega) or TransIT424 LT1 (Mirus Bio). RNA PAMP transfections were done using the TransIT-mRNA 425 Transfection kit (Mirus Bio). The siRNA transfections were done using Lipofectamine 426 RNAiMax (Invitrogen). siRNAs directed against 14-3-3ع (Dharmacon-L-017302-02-0005), 427 UFL1 (Qiagen-SI04371318) or non-targeting AllStars negative control siRNA (Qiagen428 1027280) were transfected into 293T cells (25 pmol of siRNA; final concentration of 0.0125 $429 \mu \mathrm{M}$ ) or NHDF cells (250 pmol of siRNA; final concentration of $0.25 \mu \mathrm{M}$ ). Media was 430 changed 4-24 hours post-transfection, and cells were incubated for 36-48 h post431 transfection prior to each experimental treatment. IFN- $\beta$-promoter luciferase assays were 432 performed as previously described at 18-24 hours post treatment and normalized to the 433 Renilla luciferase transfection control (33). 
435 ELISA. IFN- $\beta$ ELISAs were performed using Human IFN-beta DuoSet (R\&D Systems)

436 with supernatants collected from cultured cells.

Generation of KO cell lines. UFM1 KO 293T cells were generated by CRISPR/Cas9, using two guides targeting exon 2 and 3 , similar to others, as we have done previously $(15,30)$.

RNA analysis. Total cellular RNA was extracted using the RNeasy Plus mini kit (Qiagen). RNA was then reverse transcribed using the iScript cDNA synthesis kit (BioRad), as per 444 the manufacturer's instructions. The resulting cDNA was diluted 1:3 in ddH2O. RT-qPCR 445 was performed in triplicate using the Power SYBR Green PCR master mix (Thermo446 Fisher) and QuantStudio 6 Flex RT-PCR system. Oligonucleotide sequences for qPCR 447 are available upon request.

RNA-seq. WT and UFM1 KO 293T cells were mock or SenV-infected (18 h) and harvested in biological duplicate, followed by total RNA extraction via TRIzol reagent 451 (Thermo Fisher Scientific). Sequencing libraries were prepared using the KAPA Stranded mRNA-Seq Kit (Roche) and sequenced on an Illumina Novaseq 6000 with 50 bp pairedend reads (>20 million reads per sample) in an $\mathbf{S 1}$ flow cell by the Duke University Center 454 for Genomic and Computational Biology.

455 RNA-seq data was processed using the TrimGalore toolkit (66) which employs Cutadapt 456 (67) to trim low-quality bases and Illumina sequencing adapters from the 3' end of the 457 reads. Only reads that were 20nt or longer after trimming were kept for further analysis. 458 Reads were mapped to the GRCh38v93 version of the human genome and transcriptome 459 (68) using the STAR RNA-seq alignment tool (69). Reads were kept for subsequent 460 analysis if they mapped to a single genomic location. Gene counts were compiled using 461 the HTSeq tool (70). Only genes that had at least 10 reads in any given library were used 462 in subsequent analysis. Normalization and differential expression was carried out using 463 the DESeq2 (71) Bioconductor (72) package with the $\mathrm{R}$ statistical programming 464 environment. The false discovery rate was calculated to control for multiple hypothesis 465 testing. Gene set enrichment analysis (73) was performed to identify gene ontology terms 466 and pathways associated with altered gene expression for each of the comparisons 467 performed. All RNA-seq data are deposited in the GEO database under GSE186287. 
469 Immunoblotting. Cells were lysed in a modified radioimmunoprecipitation assay (RIPA) 470 buffer (10 mM Tris [pH 7.5], $150 \mathrm{mM} \mathrm{NaCl}, 0.5 \%$ sodium deoxycholate, and 1\% Triton X471 100) supplemented with protease inhibitor cocktail (Sigma) and Halt Phosphatase Inhibitor 472 (Thermo-Fisher), and post-nuclear lysates were isolated by centrifugation. Quantified 473 protein (between $5-15 \mu \mathrm{g}$ ) was resolved by SDS/PAGE, transferred to nitrocellulose or 474 polyvinylidene difluoride (PVDF) membranes in a $25 \mathrm{mM}$ Tris-192 mM glycine-0.01\% SDS 475 buffer. Membranes were stained with Revert 700 total protein stain (LI-COR Biosciences) 476 and then blocked in 3\% BSA in Tris-buffered saline containing 0.01\% Tween-20 (TBS-T). 477 After washing with PBS-T or TBS-T (for phosphoproteins) buffer, following incubation with 478 primary antibodies, membranes were incubated with species-specific horseradish 479 peroxidase-conjugated antibodies (Jackson ImmunoResearch, 1:5000) or fluorescent 480 secondaries (LI-COR Biosciences), followed by treatment of the membrane with Clarity 481 Western ECL substrate (BioRad) and imaging on a LICOR Odyssey FC. The following 482 antibodies were used for immunoblotting: R-anti-SenV (MBL, 1:1000), M-anti-Tubulin 483 (Sigma, 1:1000), R-anti-GAPDH (Cell Signaling Technology, 1:1000), R-anti-p-IRF3 (Cell 484 Signaling Technology, 1:1000), R-anti-IRF3 (Cell Signaling Technology, 1:1000), R-anti485 UFL1 (Novus Biologicals, 1:1000), R-anti-UFM1 (Abcam, 1:1000), anti-RIG-I (M486 AdipoGen, R-Abcam, 1:1000), R-anti-14-3-3e (Cell Signaling Technology, 1:1000), M-anti487 Flag M2 (Sigma, 1:1000), anti-Flag-HRP (Sigma, 1:1000-1:5000), R-anti-Flag (Sigma, 488 1:1000), anti-HA (M- and R-Sigma, 1:1000), and anti-Myc (M-Santa Cruz or R-Cell 489 Signaling Technology, 1:1000).

491 Immunoprecipitation. Cells were lysed in RIPA buffer with or without $10 \%$ glycerol. 492 Quantified protein (between 100-500 $\mu \mathrm{g}$ ) was incubated with protein-specific, isotype 493 control antibody (R-Cell Signaling Technology or M-Thermo Fisher), or anti-Flag M2 494 magnetic beads (Sigma), in lysis buffer either at room temperature for $2 \mathrm{~h}$ or at $4^{\circ} \mathrm{C}$ 495 overnight with head over tail rotation. The lysate/antibody mixture was then incubated with 496 Protein G Dynabeads (Invitrogen) for $1 \mathrm{~h}$. Beads were washed 3X in PBS or RIPA buffer 497 and eluted in 2X Laemmli Buffer (BioRad) with or without 5\% 2-Mercaptoethanol at $95^{\circ} \mathrm{C}$ 498 for $5 \mathrm{~min}$. Proteins were resolved by SDS/PAGE and immunoblotting, as above.

500 Subcellular membrane fractionation. Membrane fractionation was performed as 501 previously described $(7,12,38,74)$. Cells were lysed in hypotonic buffer $(10 \mathrm{mM}$ Tris-HCL 502 (pH 7.5), $10 \mathrm{mM} \mathrm{KCl}$, and $5 \mathrm{mM} \mathrm{MgCl}_{2}$ supplemented with protease inhibitor cocktail) for 
50310 minutes on ice followed by 20 passages through a 20 -guage needle. Nuclei and 504 unbroken cells were removed by centrifugation at $1000 \mathrm{xg}$ for $5 \mathrm{~min}$ at $4^{\circ} \mathrm{C}$. The resulting 505 supernatants were mixed thoroughly with $72 \%$ sucrose and overlayed with $55 \%$ sucrose, 506 followed by 10\% sucrose, all in low-salt buffer (2 nM EDTA, 20 nM HEPES (pH 8.0), 150 $507 \mathrm{mM} \mathrm{NaCl}, 0.1 \%$ SDS, $1 \%$ Triton X-100). The gradients were subjected to centrifugation at 50838,000 RPM in a Beckman SW41 Ti Rotor for $14 \mathrm{~h}$ at $4^{\circ} \mathrm{C} .1 \mathrm{~mL}$ fractions were collected 509 using a BioComp piston gradient fractionator and resulting fractions were divided in half 510 and mixed with 2 parts $100 \%$ methanol and precipitated overnight at $-80^{\circ} \mathrm{C}$. Protein pellets 511 were collected by centrifugation and resuspended in 2X Laemmli buffer and heated for 5 $512 \mathrm{~min}$ at $95^{\circ} \mathrm{C}$ for immunoblot analysis. $10 \%$ pre-fractionated cells from each condition were 513 collected as the input.

515 Semi-denaturing detergent agarose gel electrophoresis. SDD-AGE was performed as 516 described $(45,46)$. Briefly, crude mitochondria (P5 fraction) were isolated from an equal 517 number of WT or UFM1 KO 293T cells that were mock or SenV infected (12 h), 518 resuspended in hypotonic buffer (10 mM Tris, $\mathrm{pH} 7.5,10 \mathrm{mM} \mathrm{KCl}, 1.5 \mathrm{mM} \mathrm{MgCl}_{2}$, and 0.5 519 mM EDTA). Resulting samples were split and 2X SDD-AGE sample buffer (0.5X TBE, $52010 \%$ glycerol, 2\% SDS, $0.2 \mathrm{mM}$ Bromophenol Blue) buffer with or without 5\% 2521 Mercaptoethanol was added, and samples were loaded onto a vertical $1.5 \%$ agarose gel. 522 Electrophoresis was performed with a constant voltage of $70 \mathrm{~V}$ at $4{ }^{\circ} \mathrm{C}$ in SDD-AGE 523 running buffer ( $1 \mathrm{X}$ TBE and $0.1 \%$ SDS). Gels were transferred onto a nitrocellulose 524 membrane overnight on ice at $25 \mathrm{~V}$. Membranes were fixed in $0.25 \%$ glutaraldehyde in 525 PBS and immunoblotting was performed as usual. $15 \%$ of the SDD-AGE samples were 526 reserved for input.

528 Quantification of immunoblots. Immunoblots imaged using the LICOR Odyssey FC 529 were quantified by ImageStudio software, and raw values were normalized to relevant 530 controls for each antibody. Phosphoprotein values were normalized to Tubulin and 531 displayed as the percentage of signal from WT. Relative membrane association of UFL1 532 was quantified as the ratio of UFL1 to Cox-1 in fraction 1 normalized to total protein levels 533 of UFL1 in the input and displayed as the percentage of UFL1 membrane association 534 normalized to mock values. 
536 Statistical analysis. Student's unpaired t-test, one-way ANOVA, or two-way ANOVA

537 were implemented for statistical analysis of the data followed by appropriate post-hoc test

538 (as indicated) using GraphPad Prism software. Graphed values are presented as mean \pm

539 SD or SEM ( $n=3$ or as indicated); ${ }^{*} p \leq 0.05,{ }^{* *} p \leq 0.01$, and ${ }^{* * *} p \leq 0.001$.

\section{Acknowledgments}

543 We thank colleagues who provided reagents (see Methods), the Duke Functional

544 Genomics Core Facility, the Duke University Center for Genomic and Computational 545 Biology, the Duke Genomic Analysis and Bioinformatics Shared Resource and Dr. Wei 546 Chen for performing RNA-seq analysis, Dr. Madhuvanthi Vijayan and Boyoung Michelle 547 Kim for generation and initial characterization of plasmids, Dr. Matthew Thompson for 548 input in RNA-seq sample preparation and data processing, and Horner lab members for 549 useful discussion. This work was supported by funds from Burroughs Wellcome Fund, 550 National Institutes of Health: R21Al144380, R01Al155512, T32-CA009111, and an 551 American Cancer Society Postdoctoral fellowship 131321-PF-17-188-01-MPC.

553 Author Contributions: Conceptualization: D.L.S., D.C.B., and S.M.H.; Investigation: 554 D.L.S, D.C.B., and M.P.; Formal analysis: D.L.S., D.C.B., K.A.M., and S.M.H.; Writing 555 original draft: D.L.S. and S.M.H.; Writing - review and editing: D.C.B., D.L.S., and S.M.H. 556 All authors read the manuscript and provided comments. Funding acquisition: S.M.H.

558 Competing Interest Statement: The authors declare that they have no conflicts of 559 interest with the contents of this article. The content is solely the responsibility of the 560 authors and does not necessarily represent the official views of the National Institutes of 561 Health. 
563 Figure Legends

564 Figure 1. The ufmylation activity of UFL1 promotes RIG-I signaling.

565 A) IFN- $\beta$-promoter reporter luciferase expression (rel. to CMV-Renilla) from 293T cells 566 expressing vector, Flag-UFL1, or Flag-RIG-I, followed by mock or SenV infection (18 h) or 567 in B) 293 T cells transfected with vector (Vec) or Flag-UFL1, followed by mock or HCV 568 PAMP RNA transfection (24 h). C) RT-qPCR analysis (rel. to GAPDH) of RNA extracted 569 from 293T cells transfected vector or Flag-UFL1 that were treated with IFN- $\beta$ (18 h). D) 570 Immunoblot analysis of p-IRF3 following siRNA transfection along with expression of 571 vector or Flag-UFL ${ }^{\text {siR }}$, which has point mutations in the siRNA seed sequence.

572 Quantification of p-IRF3/Tubulin is shown on the right. E) RT-qPCR analysis (rel. to 18S) 573 of RNA extracted from primary neonatal human dermal fibroblasts (NHDFs) transfected 574 with either siCTRL or siUFL1 followed by mock or SenV infection (8 h). F) ELISA for IFN$575 \beta$ of supernatants harvested from NHDFs transfected with siCTRL or siUFL1 and infected 576 with SenV for the indicated times. G) Relative IFN- $\beta$-promoter reporter luciferase 577 expression (rel. to CMV-Renilla) from 293T cells expressing indicated constructs followed 578 by mock or SenV infection (12-18 h), with results graphed as relative SenV fold change 579 for each. H) Diagram of UFL1 truncation constructs and corresponding ufmylation activity 580 represented as the mean of mock and SenV values normalized to WT. For A) mean -/+ $581 \mathrm{SD}, \mathrm{n}=3$ technical replicates and representative of $n=3$ independent experiments. For all 582 others, mean $-/+$ SEM, $n=3$ or $n=5(1 G)$ biological replicates. ${ }^{*} p \leq 0.05,{ }^{* *} p \leq 0.01$, and $583{ }^{* * *} p \leq 0.001$ determined by two-way ANOVA followed by Šidák's multiple comparisons 584 test $(B, E)$, Student's t-test $(C, D, F)$, or one-way ANOVA followed by Dunnett's multiple 585 comparisons test $(\mathrm{G})$.

Figure 2. The ufmylation machinery proteins positively regulate RIG-I signaling.

588 A) IFN- $\beta$-promoter reporter luciferase expression (rel. to CMV-Renilla) from 293T cells 589 expressing vector or Flag-UFM1, followed by mock or SenV infection (18 h) or in B) WT 590 or CRISPR/CAS9 UFM1 KO 293T cells transfected with vector (Vec) or Flag-UFM1 (for $591 \mathrm{KO}$ ), followed by mock or SenV infection (18 h). C) ELISA for IFN- $\beta$ of supernatants 592 harvested from WT or CRISPR/CAS9 UFM1 KO 293T cells that were SenV infected (18 593 h). D) Diagram of UFM1 conjugation. E) Relative IFN- $\beta$-promoter reporter luciferase 594 expression (rel. to CMV-Renilla) from 293T cells expressing indicated constructs followed 595 by mock or SenV infection $(18 \mathrm{~h}$ ), with results graphed as relative SenV fold change for 596 each. Represented as mean $-/+$ SEM, $n=3$ biological replicates. ${ }^{*} p \leq 0.05,{ }^{* *} p \leq 0.01$, and 
${ }^{* * *} p \leq 0.001$ determined by two-way ANOVA followed by Tukey's multiple comparisons

598 test $(A-B)$, Student's t-test $(C)$, or one-way ANOVA followed by Dunnett's multiple comparisons test $(\mathrm{E})$.

600

Figure 3. UFM1 is required for the RIG-I driven transcriptional response.

602 RNA-seq analysis WT or UFM1 KO 293T cells following mock or SenV infection (18 h).

603 A) Gene set enrichment analysis of negatively regulated differentially expressed genes in 604 SenV-infected 293T cells represented by normalized enrichment score (UFM1 KO / WT).

605 B) Volcano plot of differentially expressed genes (adj $\mathrm{P}<0.01$ ) shown in grey, with ISGs 606 shown in red, in SenV-infected 293T cells (UFM1 KO / WT). C) Heatmap of the effect of 607 UFM1 KO on the fold change of the 50 most induced IFN and ISGs (UFM1 KO / WT) 608 following SenV infection (adj $P<0.01$ ).

609

Figure 4. UFL1 is recruited to intracellular membranes and interacts with 14-3-3e and RIG-I during RNA virus infection.

612 A) Immunoblot analysis of inputs and subcellular membrane flotation of 293T cell extracts

613 that were mock or SenV-infected $(4 \mathrm{~h})$ followed by sucrose gradient fractionation, with

614 fraction numbers indicated from the top of the gradient (1) to bottom (8). Fractionation

615 controls, GAPDH for cytosol and Cox-I for membranes, are indicated and reveal that the

616 membranes are localized to fraction \#1. Relative quantification of the ratio of UFL1 to a 617 membrane marker (Cox-I) in fraction 1 normalized to total protein levels in inputs are 618 shown on the right. B) Immunoblot analysis of anti-Flag immunoprecipitated extracts and 619 inputs from 293T cells expressing Myc-14-3-3e and Flag-UFL1 that were mock- or SenV620 infected (4 h), with relative quantification on right. C) Immunoblot analysis of anti-Flag 621 immunoprecipitated extracts and inputs from 293T cells expressing Myc-UFL1 and Flag622 RIG-I that were mock- or SenV-infected (4 h), with relative quantification with IP values 623 normalized to inputs values on right. D) Immunoblot analysis of anti-RIG-I 624 immunoprecipitated (or anti-lgG) extracts and inputs from 293T cells that were mock- or 625 SenV-infected (4 h), with relative quantification with IP values normalized to inputs values 626 on right. E) Immunoblot analysis of anti-Flag immunoprecipitated extracts and inputs from 627 293T cells expressing Myc-UFL1 and Flag-RIG-I constructs that were mock- or SenV628 infected $(4 \mathrm{~h}$ ), with results quantified as relative fold change (SenV to Mock) for each. The 629 graphs are represented as the mean -/+ SEM, $n=3$ (A-B, D-E) or $n=4$ (C) biological 
630 replicates and ${ }^{*} p \leq 0.05,{ }^{* *} p \leq 0.01$, and ${ }^{* * *} p \leq 0.001$ determined by Student's t-test (A-D)

631 or one-way ANOVA followed by Dunnett's multiple comparisons test $(E)$.

632

633 Figure 5. UFL1 interaction with RIG-I requires 14-3-3 $\varepsilon$ and ufmylation.

634 A) Immunoblot analysis of anti-HA immunoprecipitated extracts and inputs from 293T cells

635 transfected with siCTRL or si14-3-3e followed by SenV infection (4h). B) Immunoblot of 636 anti-HA immunoprecipitated extracts and inputs from 293T WT or UFM1 KO cells 637 transfected with HA-RIG-I and Flag-UFL1. C) Immunoblot of anti-Flag immunoprecipitated 638 extracts and inputs from 293T WT or UFM1 KO cells transfected with Flag-UFL1 and Myc639 14-3-3 $\varepsilon$. In (A-C), SenV infection was for 4 hours, and relative quantification is shown on 640 the right, indicating the mean $-/+\operatorname{SEM}(A, B), n=3(A, B)$ biological replicates. For (C) 641 values shown are SD of IP values adjusted for input expression, with $n=2$ biological 642 replicates. ${ }^{*} p \leq 0.05,{ }^{* *} p \leq 0.01$, and ${ }^{* * *} p \leq 0.001$ determined by Student's t-test.

644 Figure 6. Ufmylation promotes RIG-I interaction with 14-3-3\& for MAVS activation.

645 A) Immunoblot of anti-Flag immunoprecipitated extracts and inputs from 293T cells 646 transfected with siCTRL or siUFL1 and indicated constructs. B) Immunoblot of anti-Flag 647 immunoprecipitated extracts and inputs from 293T WT or UFM1 KO cells. C) Immunoblot 648 of anti-Myc immunoprecipitated extracts from 293T WT or UFM1 KO cells. D) 293T WT or 649 UFM1 KO were mock or SenV-infected (12 h). Immunoblotting shows endogenous MAVS 650 in input samples and MAVS aggregation from P5 fractions, in the presence or absence of 651 denaturing reagent ( $\beta$-mercaptoethanol). SenV infection was for $4 \mathrm{~h}(\mathrm{~A}-\mathrm{C})$ or $12 \mathrm{~h}$ (D). In 652 (A-C), relative quantification of indicated protein in the IP is shown on the right; in (D) SDD653 AGE MAVS values are normalized to corresponding SDS-PAGE values. Graphs show the 654 mean $-/+$ SEM for $n=3$ biological replicates. ${ }^{*} p \leq 0.05$, ${ }^{* *} p \leq 0.01$, and ${ }^{* * *} p \leq 0.001$ 655 determined by Student's t-test.

657 Figure S1. The domains of UFL1 that form UFM1 conjugates in cells.

658 Quantification of immunoblots from 293T cells expressing indicated Flag-UFL1

659 constructs or vector followed by mock or SenV infection (18 $\mathrm{h}$ ) represented as the ratio 660 of UFM1 conjugates (approximately 25-50 kDa) to Flag-UFL1 expression in each lane 
661 normalized to loading control with WT Flag-UFL1 mock set to 100. Graph indicates the

662 mean -/+ SEM for $n=3$ biological replicates.

663

664 Fig. S2. Transcriptional response of genes negatively regulated by UFM1.

665 RNA-seq analysis of WT versus UFM1 KO 293T cells showing the gene set enrichment

666 analysis (top 10 categories) of negatively regulated differentially expressed genes

667 represented by normalized enrichment score to identify gene ontology terms and

668 pathways associated with altered gene expression for each of the comparisons

669 performed (adj $P<0.01)$.

670

671 Dataset S1. Differential expression analysis from RNA-seq analysis for UFM1 KO / WT

672 293T cells

673 Table S1.1: UFM1 KO / WT Mock

674 Table S1.2 UFM1 KO / WT SenV (18 h)

675

676 Dataset S2. Gene Set Enrichment Analysis for UFM1 KO / WT 293T cells

677 Table S2.1: UFM1 KO / WT Mock- negative direction

678 Table S2.2 UFM1 KO / WT SenV (18 h)- negative direction

679 Table S2.3 UFM1 KO / WT Mock- positive direction

680 Table S2.4 UFM1 KO / WT SenV (18 h)- negative direction 


\section{References}

682

683

684

685

686

687

688

689

690

691

692

693

694

695

696

697

698

699

700

701

702

703

704

705

706

707

708

709

710

711

712

713

714

715

1. D. Thoresen, et al., The molecular mechanism of RIG-I activation and signaling. Immunol Rev (2021) https:/doi.org/10.1111/imr.13022.

2. M. J. McFadden, N. S. Gokhale, S. M. Horner, Protect this house: cytosolic sensing of viruses. Current Opinion in Virology 22, 36-43 (2017).

3. J. Rehwinkel, M. U. Gack, RIG-I-like receptors: their regulation and roles in RNA sensing. Nature Reviews Immunology, 1-15 (2020).

4. C. Chiang, M. U. Gack, Post-translational Control of Intracellular Pathogen Sensing Pathways. Trends in Immunology 38, 39-52 (2017).

5. M. U. Gack, et al., TRIM25 RING-finger E3 ubiquitin ligase is essential for RIG-I-mediated antiviral activity. Nature 446, 916-920 (2007).

6. H. Oshiumi, M. Matsumoto, S. Hatakeyama, T. Seya, Riplet/RNF135, a RING finger protein, ubiquitinates RIG-I to promote interferon-beta induction during the early phase of viral infection. J. Biol. Chem. 284, 807-817 (2009).

7. H. M. Liu, et al., The Mitochondrial Targeting Chaperone 14-3-3ع Regulates a RIG-I Translocon that Mediates Membrane Association and Innate Antiviral Immunity. Cell Host \& Microbe 11, 528-537 (2012).

8. H. Oshiumi, Recent Advances and Contradictions in the Study of the Individual Roles of Ubiquitin Ligases That Regulate RIG-I-Like Receptor-Mediated Antiviral Innate Immune Responses. Front Immunol 11, 1296 (2020).

9. Y. K. Chan, M. U. Gack, A phosphomimetic-based mechanism of dengue virus to antagonize innate immunity. Nature Immunology 17, 523-530 (2016).

10. W. Riedl, et al., Zika Virus NS3 Mimics a Cellular 14-3-3-Binding Motif to Antagonize RIGI- and MDA5-Mediated Innate Immunity. Cell Host \& Microbe 26, 493-503.e6 (2019).

11. C. Vazquez, S. M. Horner, MAVS Coordination of Antiviral Innate Immunity. J Virol 89, 6974-6977 (2015).

12. E.-H. Tam, et al., Role of the chaperone protein $14-3-3 \varepsilon$ in the regulation of influenza $A$ virus-activated beta interferon. J Virol, JVI0023121 (2021).

13. S. M. Horner, H. M. Liu, H. S. Park, J. Briley, M. Gale, Mitochondrial-associated endoplasmic reticulum membranes (MAM) form innate immune synapses and are targeted by hepatitis C virus. Proc Natl Acad Sci U S A 108, 14590-14595 (2011).

14. S. M. Horner, C. Wilkins, S. Badil, J. Iskarpatyoti, M. G. Jr, Proteomic Analysis of Mitochondrial-Associated ER Membranes (MAM) during RNA Virus Infection Reveals Dynamic Changes in Protein and Organelle Trafficking. PLOS ONE 10, e0117963 (2015). 
716

717

718

719

720

721

722

723

724

725

726

727

728

729

730

731

732

733

734

735

736

737

738

739

740

741

742

743

744

745

746

747

748

749

750

15. D. C. Beachboard, et al., The small GTPase RAB1B promotes antiviral innate immunity by interacting with TNF receptor-associated factor 3 (TRAF3). J. Biol. Chem., jbc.RA119.007917 (2019).

16. K. Tatsumi, et al., A novel type of $\mathrm{E} 3$ ligase for the Ufm1 conjugation system. J. Biol. Chem. 285, 5417-5427 (2010).

17. M. Komatsu, et al., A novel protein-conjugating system for Ufm1, a ubiquitin-fold modifier. EMBO J 23, 1977-1986 (2004).

18. S. H. Kang, et al., Two novel ubiquitin-fold modifier 1 (Ufm1)-specific proteases, UfSP1 and UfSP2. J. Biol. Chem. 282, 5256-5262 (2007).

19. Y. Wei, X. Xu, UFMylation: A Unique \& Fashionable Modification for Life. Genomics Proteomics Bioinformatics 14, 140-146 (2016).

20. J. Daniel, E. Liebau, The Ufm1 Cascade. Cells 3, 627-638 (2014).

21. L. Wang, et al., UFMylation of RPL26 links translocation-associated quality control to endoplasmic reticulum protein homeostasis. Cell Res, 1-16 (2019).

22. Z. Wang, et al., MRE11 UFMylation promotes ATM activation. Nucleic Acids Research 47, 4124-4135 (2019).

23. H. M. Yoo, et al., Modification of ASC1 by UFM1 is crucial for ERa transactivation and breast cancer development. Mol. Cell 56, 261-274 (2014).

24. B. Qin, et al., UFL1 promotes histone H4 ufmylation and ATM activation. Nat Commun 10, $1242(2019)$.

25. I. A. Gak, et al., UFMylation regulates translational homeostasis and cell cycle progression. bioRxiv, 2020.02.03.931196 (2020).

26. R. DeJesus, et al., Functional CRISPR screening identifies the ufmylation pathway as a regulator of SQSTM1/p62. eLife 5, e17290 (2016).

27. J. R. Liang, et al., A Genome-wide ER-phagy Screen Highlights Key Roles of Mitochondrial Metabolism and ER-Resident UFMylation. Cell 0 (2020).

28. C. P. Walczak, et al., Ribosomal protein RPL26 is the principal target of UFMylation. PNAS 116, 1299-1308 (2019).

29. Y. Cai, N. Singh, H. Li, Essential role of Ufm1 conjugation in the hematopoietic system. Exp. Hematol. 44, 442-446 (2016).

30. J. Kulsuptrakul, R. Wang, N. L. Meyers, M. Ott, A. S. Puschnik, A genome-wide CRISPR screen identifies UFMylation and TRAMP-like complexes as host factors required for hepatitis A virus infection. Cell Reports 34 (2021).

31. D. R. Balce, et al., UFMylation inhibits the proinflammatory capacity of interferon- $\gamma-$ activated macrophages. PNAS 118 (2021). 
32. Y.-M. Loo, et al., Viral and therapeutic control of IFN- $\beta$ promoter stimulator 1 during hepatitis C virus infection. PNAS 103, 6001-6006 (2006).

33. R. Sumpter, et al., Regulating intracellular antiviral defense and permissiveness to hepatitis C virus RNA replication through a cellular RNA helicase, RIG-I. J Virol 79, 26892699 (2005).

34. T. Saito, D. M. Owen, F. Jiang, J. Marcotrigiano, M. Gale, Innate immunity induced by composition-dependent RIG-I recognition of hepatitis C virus RNA. Nature 454, 523-527 (2008).

35. D. Simsek, et al., The Mammalian Ribo-interactome Reveals Ribosome Functional

36. M. J. McFadden, et al., Post-transcriptional regulation of antiviral gene expression by N6-

37. A. Peisley, B. Wu, H. Xu, Z. J. Chen, S. Hur, Structural basis for ubiquitin-mediated antiviral signal activation by RIG-I. Nature 509, 110-114 (2014).

38. A. Mizutani, M. Fukuda, K. Ibata, Y. Shiraishi, K. Mikoshiba, SYNCRIP, a Cytoplasmic Counterpart of Heterogeneous Nuclear Ribonucleoprotein R, Interacts with Ubiquitous Synaptotagmin Isoforms*. Journal of Biological Chemistry 275, 9823-9831 (2000).

39. E. Foy, et al., Control of antiviral defenses through hepatitis $C$ virus disruption of retinoic acid-inducible gene-I signaling. Proc. Natl. Acad. Sci. U.S.A. 102, 2986-2991 (2005).

40. E. Meylan, et al., Cardif is an adaptor protein in the RIG-I antiviral pathway and is targeted by hepatitis C virus. Nature 437, 1167-1172 (2005).

41. X.-D. Li, L. Sun, R. B. Seth, G. Pineda, Z. J. Chen, Hepatitis C virus protease NS3/4A cleaves mitochondrial antiviral signaling protein off the mitochondria to evade innate immunity. Proc Natl Acad Sci U S A 102, 17717-17722 (2005).

42. R. Lin, et al., Dissociation of a MAVS/IPS-1/VISA/Cardif-IKKepsilon molecular complex from the mitochondrial outer membrane by hepatitis C virus NS3-4A proteolytic cleavage. $J$ Virol 80, 6072-6083 (2006).

43. K. Takahasi, et al., Nonself RNA-Sensing Mechanism of RIG-I Helicase and Activation of Antiviral Immune Responses. Molecular Cell 29, 428-440 (2008).

44. H. Oshiumi, M. Miyashita, M. Matsumoto, T. Seya, A distinct role of Riplet-mediated K63Linked polyubiquitination of the RIG-I repressor domain in human antiviral innate immune responses. PLoS Pathog. 9, e1003533 (2013).

45. F. Hou, et al., MAVS Forms Functional Prion-like Aggregates to Activate and Propagate Antiviral Innate Immune Response. Cell 146, 448-461 (2011).

46. N. Zamorano Cuervo, Q. Osseman, N. Grandvaux, Virus Infection Triggers MAVS Polymers of Distinct Molecular Weight. Viruses 10 (2018). 
47. W. Zeng, et al., Reconstitution of the RIG-I pathway reveals a signaling role of unanchored polyubiquitin chains in innate immunity. Cell 141, 315-330 (2010).

48. X. Jiang, et al., Ubiquitin-induced oligomerization of the RNA sensors RIG-I and MDA5 activates antiviral innate immune response. Immunity 36, 959-973 (2012).

49. L. Wang, et al., UFMylation of RPL26 links translocation-associated quality control to endoplasmic reticulum protein homeostasis. Cell Research 30, 5-20 (2020).

50. Y. Zhang, M. Zhang, J. Wu, G. Lei, H. Li, Transcriptional regulation of the Ufm1 conjugation system in response to disturbance of the endoplasmic reticulum homeostasis and inhibition of vesicle trafficking. PLOS ONE 7, e48587 (2012).

51. C. Kwak, et al., Contact-ID, a tool for profiling organelle contact sites, reveals regulatory proteins of mitochondrial-associated membrane formation. PNAS 117, 12109-12120 (2020).

52. Y. Merbl, P. Refour, H. Patel, M. Springer, M. W. Kirschner, Profiling of ubiquitin-like modifications reveals features of mitotic control. Cell 152, 1160-1172 (2013).

53. L. Wang, et al., UFMylation of RPL26 links translocation-associated quality control to endoplasmic reticulum protein homeostasis. Cell Res. 30, 5-20 (2020).

54. M. Hochstrasser, Origin and function of ubiquitin-like proteins. Nature 458, 422-429 (2009).

55. K. N. Swatek, D. Komander, Ubiquitin modifications. Cell Research 26, 399-422 (2016).

56. A. J. Muslin, J. W. Tanner, P. M. Allen, A. S. Shaw, Interaction of 14-3-3 with Signaling Proteins Is Mediated by the Recognition of Phosphoserine. Cell 84, 889-897 (1996).

57. Y. Aghazadeh, V. Papadopoulos, The role of the 14-3-3 protein family in health, disease, and drug development. Drug Discovery Today 21, 278-287 (2016).

58. A. Aitken, Post-translational modification of $14-3-3$ isoforms and regulation of cellular function. Seminars in Cell \& Developmental Biology 22, 673-680 (2011).

59. J.-P. Lin, Y.-K. Fan, H. M. Liu, The 14-3-3n chaperone protein promotes antiviral innate immunity via facilitating MDA5 oligomerization and intracellular redistribution. PLoS Pathog. 15, e1007582 (2019).

60. K. Onomoto, K. Onoguchi, M. Yoneyama, Regulation of RIG-I-like receptor-mediated

61. G. Liu, et al., ISG15-dependent activation of the sensor MDA5 is antagonized by the SARS-CoV-2 papain-like protease to evade host innate immunity. Nature Microbiology, 112 (2021).

62. M. Yoneyama, et al., Shared and Unique Functions of the DExD/H-Box Helicases RIG-I, MDA5, and LGP2 in Antiviral Innate Immunity. The Journal of Immunology 175, 28512858 (2005). 
823 63. B. Fredericksen, et al., Activation of the Interferon- $\beta$ Promoter During Hepatitis C Virus RNA Replication. Viral Immunology 15, 29-40 (2002).

64. T. Saito, et al., Regulation of innate antiviral defenses through a shared repressor domain in RIG-I and LGP2. PNAS 104, 582-587 (2007).

65. F. A. Ran, et al., Genome engineering using the CRISPR-Cas9 system. Nat Protoc 8, 2281-2308 (2013).

66. Babraham Bioinformatics - Trim Galore! (October 12, 2021).

67. M. Martin, Cutadapt removes adapter sequences from high-throughput sequencing reads. EMBnet.journal 17, 10-12 (2011).

68. P. J. Kersey, et al., Ensembl Genomes: an integrative resource for genome-scale data from non-vertebrate species. Nucleic Acids Res 40, D91-97 (2012).

69. A. Dobin, et al., STAR: ultrafast universal RNA-seq aligner. Bioinformatics 29, 15-21 (2013).

70. HTSeq: Analysing high-throughput sequencing data with Python - HTSeq 0.13 .5 documentation (October 12, 2021).

71. M. I. Love, W. Huber, S. Anders, Moderated estimation of fold change and dispersion for RNA-seq data with DESeq2. Genome Biology 15, 550 (2014).

840 72. W. Huber, et al., Orchestrating high-throughput genomic analysis with Bioconductor. Nat Methods 12, 115-121 (2015).

73. V. K. Mootha, et al., PGC-1alpha-responsive genes involved in oxidative phosphorylation are coordinately downregulated in human diabetes. Nat Genet 34, 267-273 (2003).

844 74. H. M. Liu, et al., SYNCRIP (synaptotagmin-binding, cytoplasmic RNA-interacting protein) is 


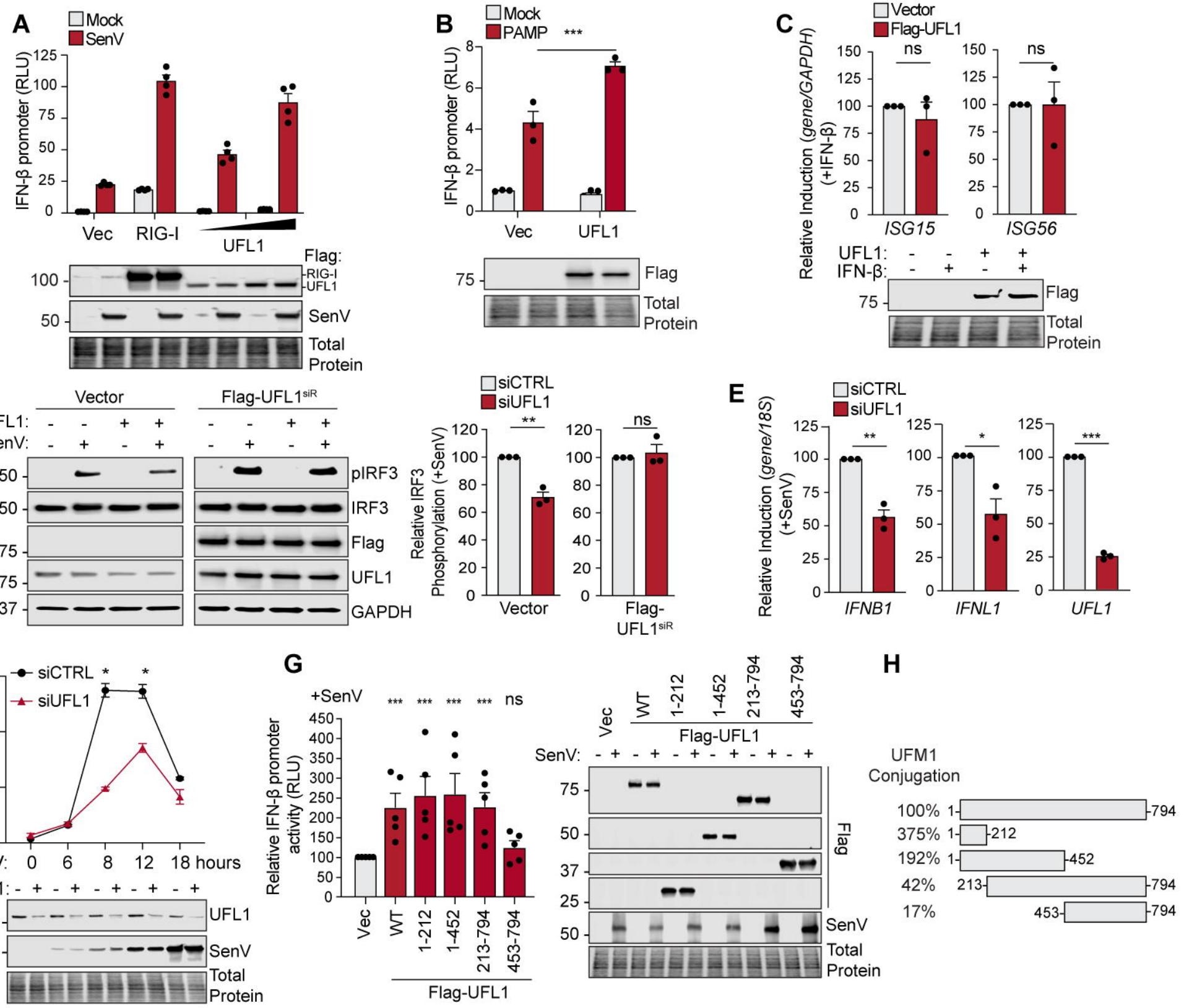


Response to Type I IFN Defense/Response to Virus Innate Immune Response

Response to Virus

Negative Regulation of Viral Process

Cytokine-Mediated Signaling Pathway Negative Regulation of Viral Genome Replication Regulation of Type I IFN Production Response to IFNy Defense/Response to Other Organism -

B

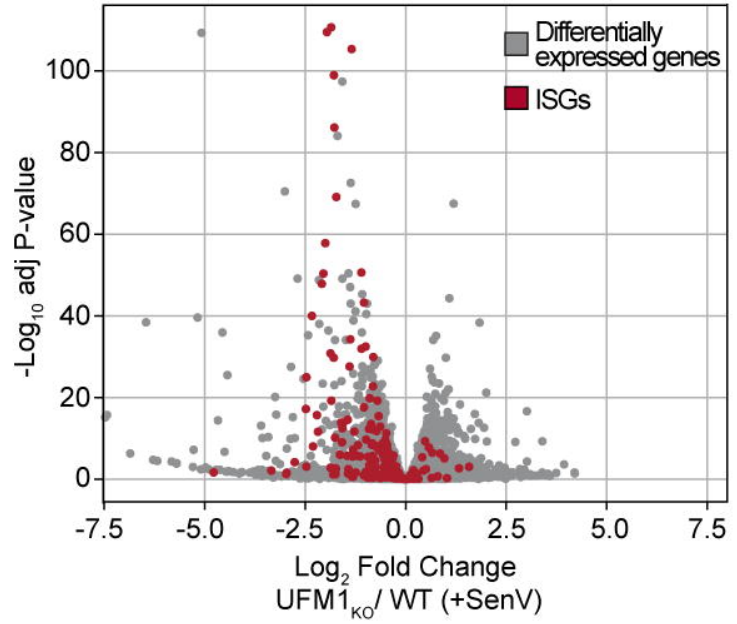

C

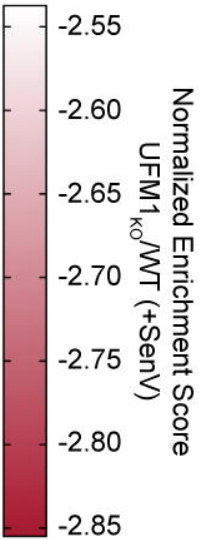

RARRES3

GBP5

ODF3B

IFNB1

TRIM22

IFNL1.

RSAD2

TRANK1 .

BST2

DDX58

IFI44

IFIT2

OASL

IFITM3

ISG20

IFIH1

IFIT3

GPB1

IFI44L

HERC5

$\mathrm{DHX58}$

OAS2

IFITM1

IFIT1

$\log _{2}$ Fold Change

$\mathrm{UFM}_{\mathrm{KO}} \mathrm{NT}$ (+SenV)

$-4.5-4.0-3.5-3.0-2.5-2.0-1.5$ 


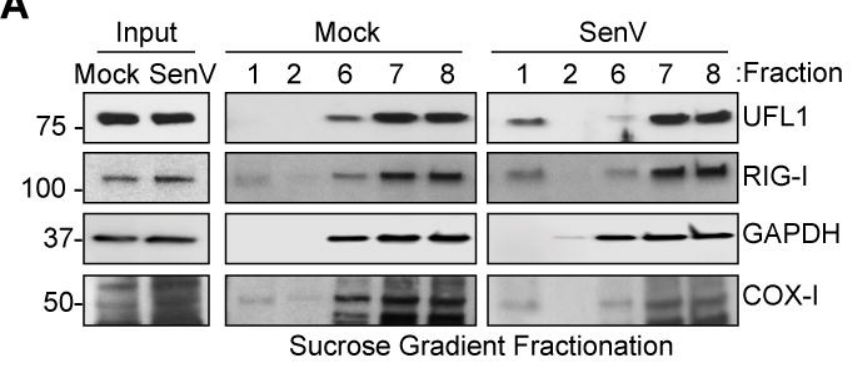

Sucrose Gradient Fractionation

\section{C}

$\frac{\text { Mock }}{++} \frac{\text { SenV }}{++}$

Flag-RIG-I:
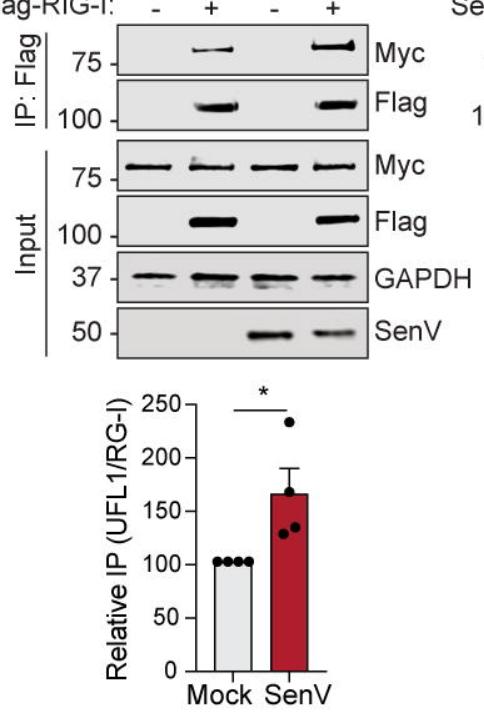

IP: IgG RIG-I

SenV:
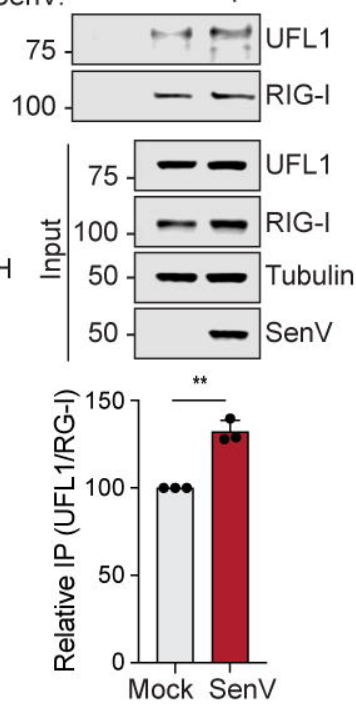

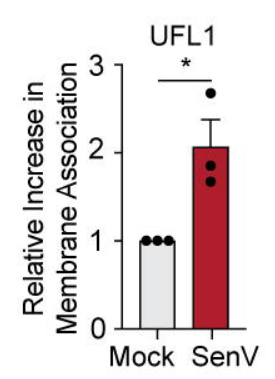

B

Mock SenV
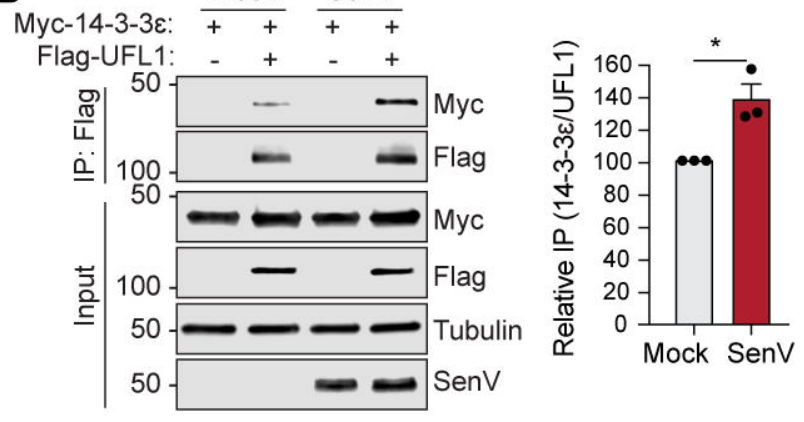

E

Flag-RIG-I

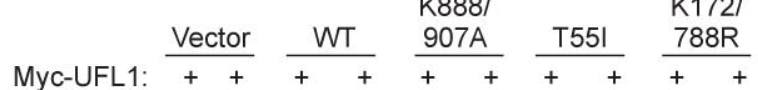
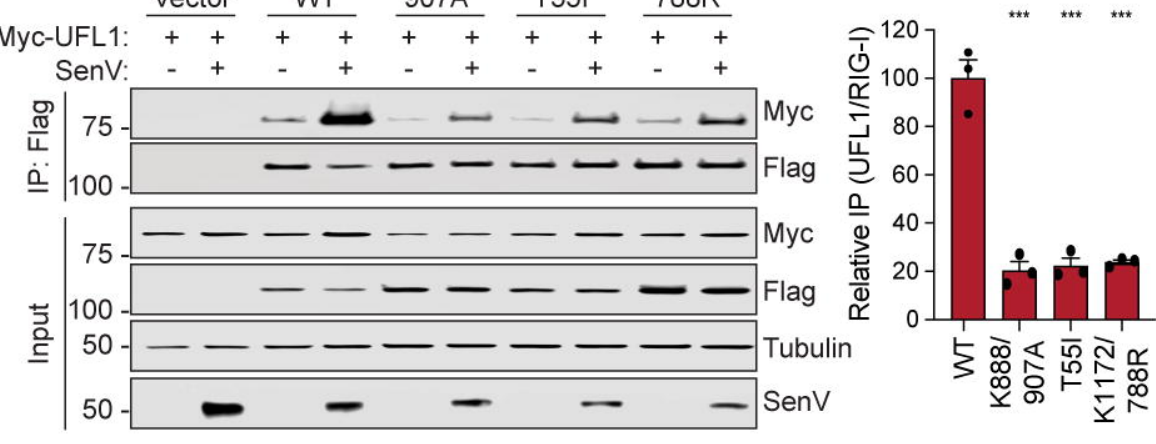
$\mathbf{A}$

siCTRL si14-3-3ع

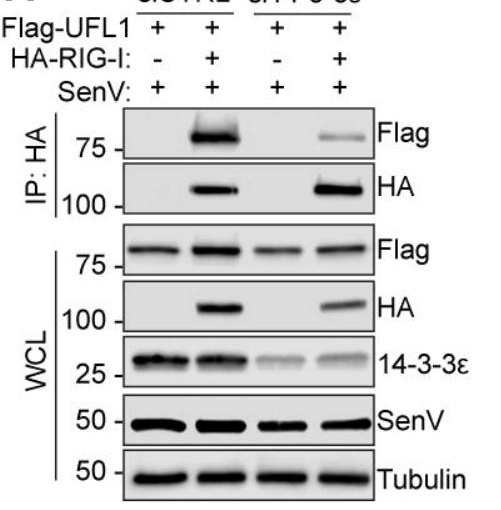

WT UFM1 $1_{\text {ко }} \quad$ C

WT UFM $1_{\text {ко }}$

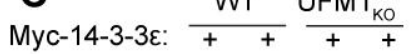

Flag-UFL1:

SenV: ++++

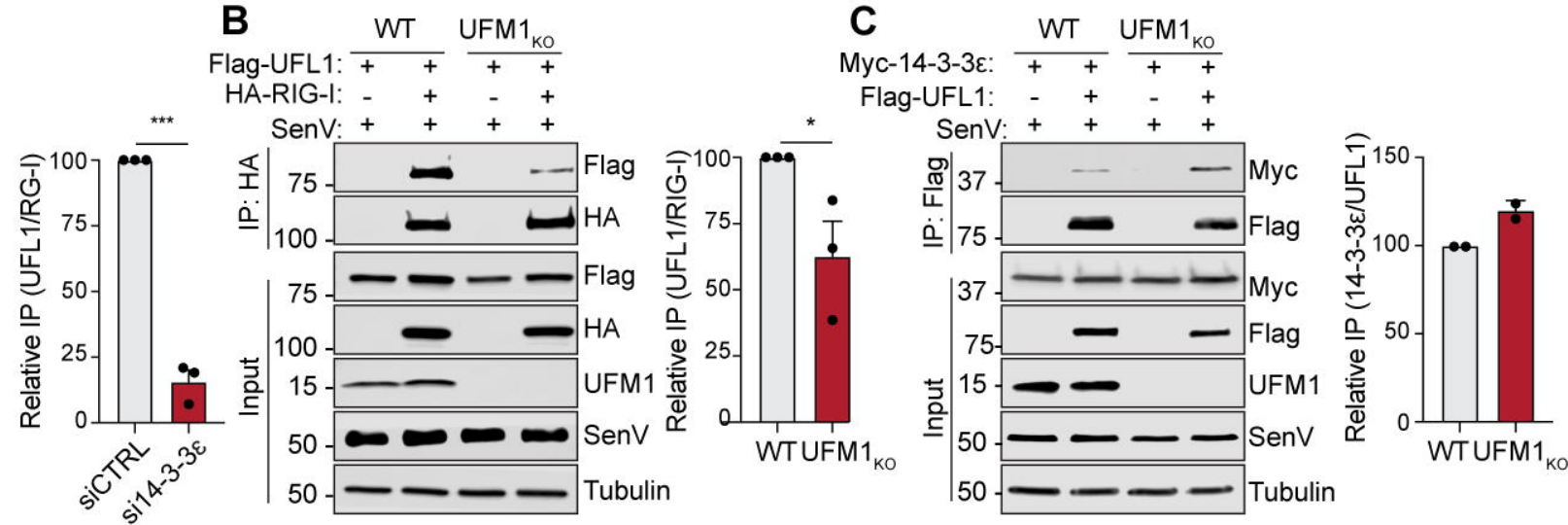

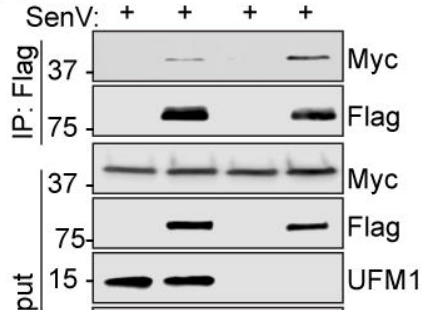

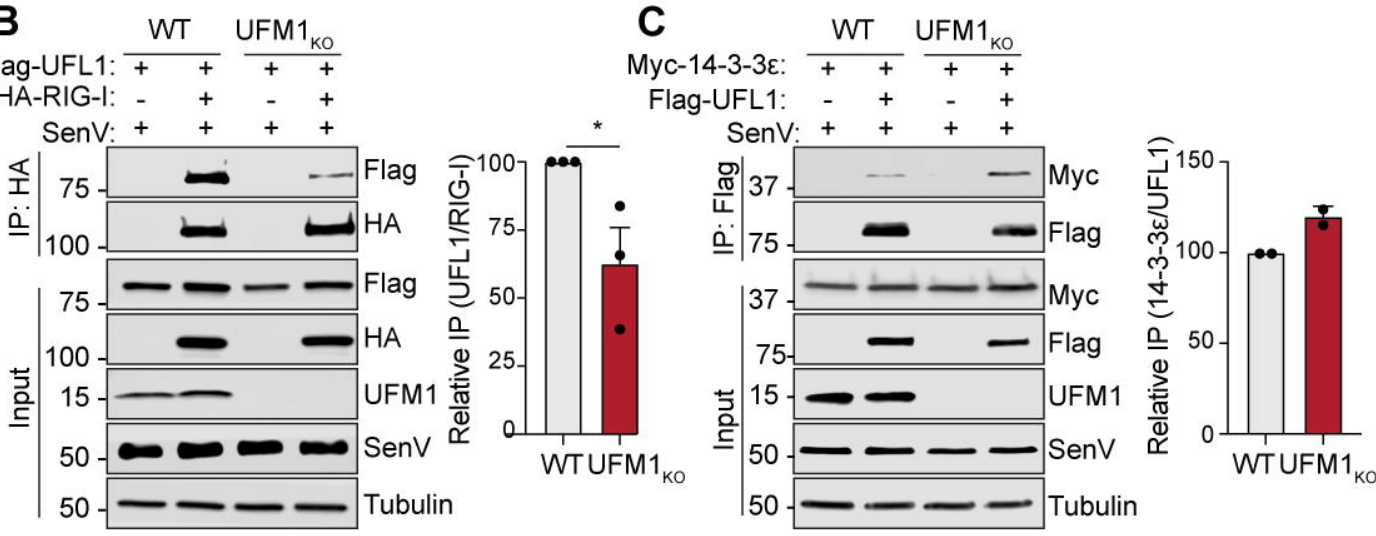


siCTRL

siUFL1

\begin{tabular}{|c|c|c|c|c|c|c|c|c|}
\hline & & & & & & & & \\
\hline 1ус-14-3- & + & + & + & + & + & + & + & + \\
\hline Flag-RIG-I & - & - & + & + & - & - & + & + \\
\hline SenV: & - & + & - & + & - & + & - & + \\
\hline
\end{tabular}
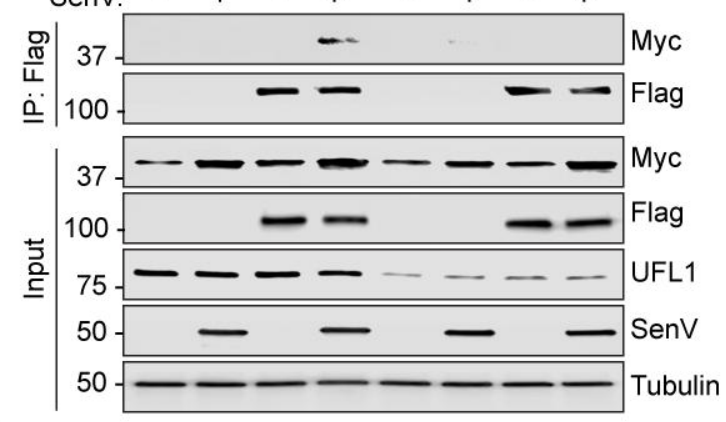

B
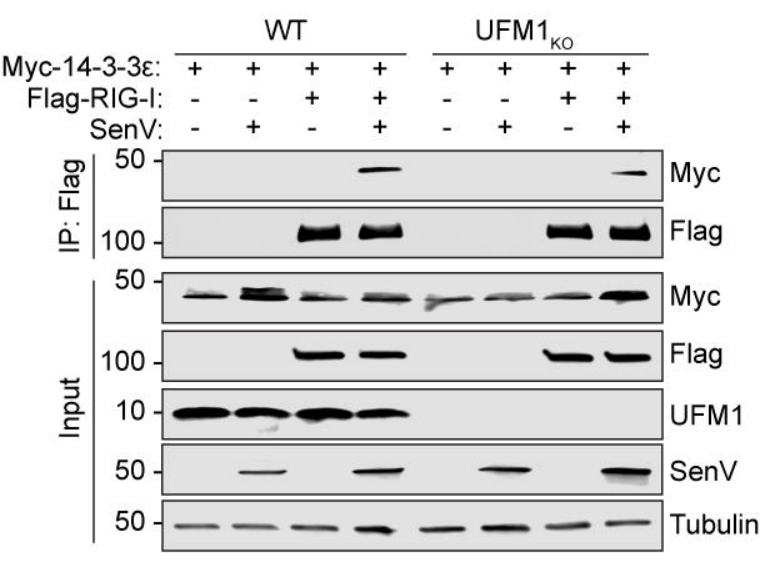
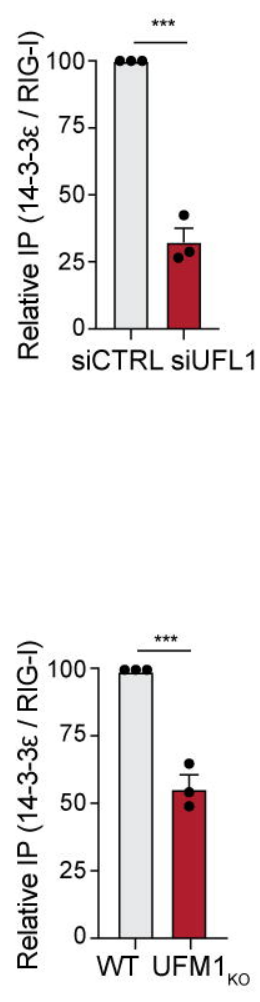

Clag-RIG-I: $_{\text {C }} \frac{\text { WT }}{+t} \frac{\text { UFM1 }}{t+}$

Myc-MAVS:
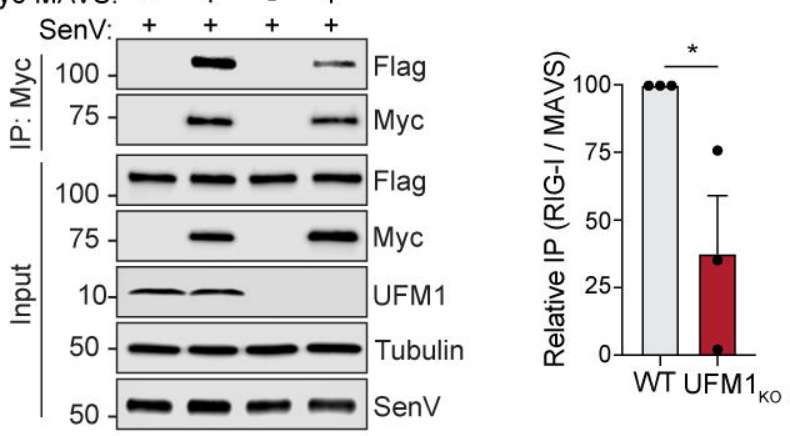

D

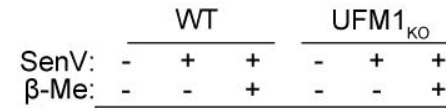

क

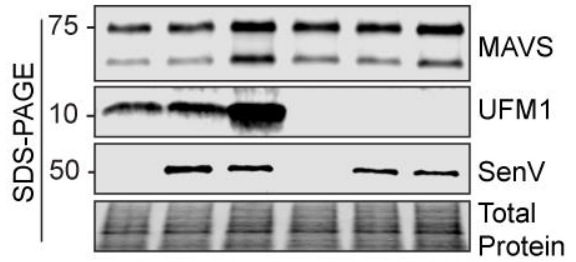

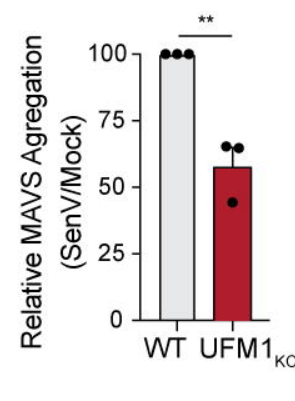




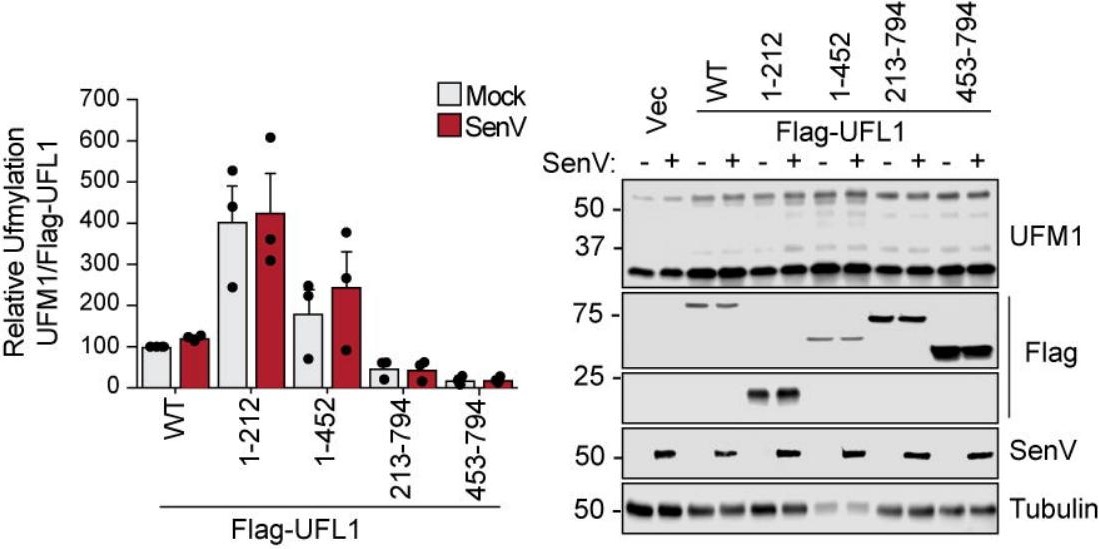


Nephron Epithelium Development Nephron Development -

Kidney Epithelium Development Renal Tubule Development Regulation of Platelet Activation -

Negative Regulation of Coagulation Mesonephric Tubule Morphogenesis Cytosolic Ribosome -

Cytosolic Large Ribosomal Subunit Ras Guanyl Nucleotide Exchange Factor - 